Boise State University

ScholarWorks

7-1-2015

Easing the Transition from Clinician to Nurse Educator: An Integrative Literature Review

Jane S. Grassley

Boise State University

Andrea Lambe

Boise State University 


\title{
Easing the Transition from Clinician to Nurse Educator: An Integrative Literature Review
}

\author{
Jane Grassley \\ Boise State University \\ Andrea Lambe \\ Boise State University
}

\section{Introduction}

Many experienced clinicians find the transition into nursing education to be difficult and discouraging (Anderson, 2009). These nurses bring strong clinical skills and a desire to make a positive difference in the lives of students, but are unprepared for the challenges they may encounter (Schoening, 2013). This integrative literature review was conducted out of a genuine concern for these expert clinicians as they transition into their first academic faculty position.

Clinicians often have expectations that are incongruent with the realities of the faculty role. They find themselves unprepared for the cultural differences between nursing practice and academic nursing education (Schoening, 2013; Siler \& Kleiner, 2001). Positive encounters with students as a preceptor differ from the stresses experienced in student interactions as their faculty (Anderson, 2009; Schriner, 2007). Clinical competence and expertise, while important, does not prepare clinicians for teaching students (Anderson, 2009); many come to their first academic appointment with no formal preparation and learn to teach in their first year (Schoening, 2013). This process of learning to teach is characterized by discomfort at being a novice again, uncertainty about how to learn to teach, and fear of failing in their new role (Cangelosi, Crocker and Sorrell, 2009).

Given the current and future faculty shortages, investment by academic nursing programs in easing the challenges experienced by clinicians new to nursing education is essential to their retention as faculty (McAllister, Oprescu, \& Jones, 2014). However, the literature documents a consistent lack of adequate mentoring and formal orientation to the academic setting (Anderson, 2009; Cangelosi et al., 2009; Santisteban \& Egues, 2014; Schoening, 2013). Therefore, an integrative review of the literature was conducted to explore the essential components of a comprehensive program to facilitate a positive experience for nurse clinicians transitioning to a faculty role.

\section{Method}

Searches of the ProQuest Central and Cinahl databases for peer-reviewed articles were conducted using mentoring or orientation, nursing education, expert clinician, and novice nurse educator (nine relevant articles) and mentoring, faculty, and nursing education as key terms (seven relevant articles). One article was replicated; hand searching provided two more articles. A total of 17 were included in this integrative review. A Google search also was conducted using the key terms of schools of nursing and faculty mentoring programs to explore current mentoring programs that schools of nursing have implemented. This search yielded five websites that were reviewed for insights into developing a faculty mentoring toolkit.

\section{Results}

Table 1 provides an overview of the literature reviewed and their insights into strategies for implementing a comprehensive orientation program to facilitate the transition of master nurse clinicians to master academic educators. The literature synthesized in this integrative review included six qualitative research studies, four that discussed strategies to facilitate the transition experience from clinician to nurse educator (Cangelosi et al., 2009; Cangelosi, 2014; Gardner, 2014; Schoening, 2013) and two that described the experience of being a mentor or mentee (White, Brannan, \& Wilson, 2010; Wilson, Brannan, \& White, 2010); a survey study of mentoring needs of new faculty (Sawatzky \& Enns, 2009); two integrative reviews of the literature related to mentoring (Nick et al., 2014) and cultivating adjunct faculty (Santisteban \& Egues, 2014); two theoretical papers discussing mentorship models (Jacobsen \& Sherrod, 2012; McAllister et al., 2014); and six descriptions of a particular orientation or 
mentoring program in a school of nursing (Baker, 2010; Berk, 2010; Blauvelt \& Spath, 2008; Hunt, Curtis, \& Sanderson, 2013; Reid, Hinderer, Jaroskinski, Mister, \& Seldomridge, 2013; Slimmer, 2012). Strategies from four universities' mentoring programs that could be accessed online were included in the review (College of Dupage [COD], 2014; University of California San Francisco [UCSF], 2014; University of North Carolina [UNC] Chapel Hill, 2014; University of Pennsylvania [UPenn], 2014). The Mentoring of Nursing Faculty Toolkit available from the National League for Nursing (NLN, 2008) and two online clinical faculty courses were also reviewed (Connecticut League of Nursing [CNL], 2014; Indiana University School of Nursing [IUSON], 2014). A review of this literature identified formal preparation for teaching, guidance navigating the academic culture, and a structured mentoring program as the essential components of a comprehensive program to facilitate clinicians' successful transition to academic nurse faculty.

\section{Discussion}

\section{Formal Preparation for Teaching}

Teaching nursing is different than practicing nursing. Many clinicians become faculty with little preparation in how to teach, a major source of stress in the first year of teaching. This was a consistent theme reported in the literature (Cangelosi et al., 2009; Gardner, 2014; Santisteban \& Egues, 2014; Schoening, 2013; White et al., 2010). To ease this transition, clinicians need formal preparation for the teaching role, which can include topics such as defining the faculty role, planning and guiding student experiences, using formative and summative evaluations, understanding ethical and legal considerations, dealing with difficult students, and incorporating simulation (Cangelosi et al., 2009; Reid et al., 2013; Schoening, 2013). Formal preparation can be offered as a face-to-face Clinical Nurse Academy (Cangelosi et al., 2009), as a 3 to 6 week online course (CNL, 2014; IUSON, 2014), or as a hybrid course such as the Eastern Shore Faculty and Mentorship Initiative (ES-FAMI), a 30-contact hour hybrid program that includes face-to-face meeting, teaching/learning simulation, and online components (Reid et al., 2013). If formal preparation is not available, new faculty development may be incorporated into a formal mentoring program (Blauvert \& Spath, 2008). In addition to formal preparation for teaching, nurse clinicians need guidance navigating the academic culture.

\section{Guidance Navigating the Academic Culture}

The clinical and academic settings have very different cultures with their own values, expectations, language, relationships, policies and procedures (McDermid, Peters, Jackson, \& Daly, 2012). Many nurse clinicians experience frustration their first year because they have limited understanding of how to navigate these differences (White et al., 2010). The literature advocates for an intentional, extended orientation process to ease their transition into the academic culture (Baker, 2010; Blauvelt \& Spath, 2008; Hunt et al., 2013; Santisteban \& Egues, 2014). Faculty orientations have traditionally been one to three day marathons in which new faculty are bombarded with a massive amount of information and little time for processing it. Baker (2010) described an alternative orientation process where the nursing college dean facilitated meetings off campus with new faculty throughout the academic year. Using a seminar approach, group members engaged in discussions and joint activities related to solving teaching and learning problems. Recommended orientation topics include discussion of the curriculum, philosophy, mission, and values of the nursing program as well as policies such as expectations related to student appearance, lateness and absences, engagement, and managing difficult student behavior (Baker, 2010; Hunt et al., 2013). Orientation should also include meeting with course leaders and colleagues (Hunt et al., 2013).

A structured orientation that includes a comprehensive faculty reference manual can facilitate new faculty's socialization into the academic culture (Santisteban \& Egues, 2014; Schoening, 2013). The essential content and format of the faculty reference manual go beyond a compilation of standard school policies. The College of DuPage (COD, 2014) includes a list of frequently asked questions in their nursing department mentor/mentee toolkit that range from practical issues like "How do I get my keys?” or "Sign up for email?" to student concerns such a "How do I document occurrences with students?” (pp. 15-16). The mentoring handbook described by Blauvelt and Spath (2008) is organized around teaching topics such as classroom management, syllabus development, test construction and analysis, and advising policies and tips. 
Santisteban and Egues (2014) developed an excellent list of orientation talking points from the literature. They included examples of topics for a new faculty orientation such as faculty role and responsibilities ("What is the chain of command when dealing with any administrator, course, faculty, or student issue?”); student-related policies (“What is the dress code and how is it reinforced?”); student evaluation ("How are clinical/classroom learning and skills evaluated?”); setting up clinical experiences (“How are optimal learner situations provided?”); clinical site information ("What orientation, testing, and training is needed prior to the start of clinical?”); clinical setting documentation ("What is the process of pre-and post- clinical instruction?) (p. 156). Other topics addressed included classroom setting access and management, simulation experiences, self-assessment, and institutional issues. Although their focus was orienting adjunct faculty, their key questions are applicable to all nursing clinical faculty. Navigating the academic culture as a novice faculty is complex and best done in the company of a mentor, the third essential component in facilitating clinicians' successful transition to the academic culture.

\section{A Structured Mentoring Program}

The importance of a formal, structured mentoring program was emphasized in the literature (Cangelosi, 2014; Gardner, 2014; NLN, 2006; White et al., 2010; Wilson et al., 2010). A mentor serves as a guide and advocate for someone entering a new role and/or a new environment (such as an expert clinician who transitions to an academic or clinical faculty role). According to the NLN Position Statement on Mentoring of Nurse Faculty, a need for mentoring occurs at all stages of a faculty member's career (NLN, 2006). However, for the purposes of this discussion, the focus will be mentoring of nurses new to the faculty role. Although mentee and protégé are used interchangeably in the literature, mentee will be used to refer to the one being mentored (NLN, 2008). The Best Practices in Academic Mentoring: A Model for Excellence developed by Nick et al. (2012) provided a framework of best practices to include in a formal mentoring program for clinicians transitioning to academic nurse educators. Best mentoring practices include achieving appropriately matched new faculty/mentor dyads, setting clear mentorship purpose and goals, solidifying the dyad relationship, integrating new faculty into the academic culture, advocating and guiding the new faculty, and mobilizing institutional resources (Nick et al., 2012, p. 3). Strategies for implementing each mentoring practice will be discussed.

Matching new faculty/mentor dyads. The literature consistently emphasized the importance of taking time to match new faculty with an appropriate mentor. Poorly matched dyads are not beneficial for either mentees or their mentors (Gardner, 2014). The strategies used to match mentees with a mentor vary. An administrator can assign mentors (Blauvelt \& Spath, 2008; COD, 2014) or be the primary mentor for all new faculty (Slimmer, 2012). In several universities a mentorship task force or a mentoring facilitator matches mentees and mentors (UCSF, 2014; UNC Chapel Hill, 2014; UPenn, 2014).

In selecting appropriate mentors, it is essential to match interests and mentee needs. At UNC Chapel Hill (2014), mentors and mentees complete an interests and strengths inventory, which the faculty mentorship task force uses to assign mentors. In another school, new faculty members are matched with mentors who have similar clinical specialties but who teach in a different semester level (Baker, 2010). To involve new faculty in the process of selecting a mentor, Berk (2010) recommended a "speed" mentoring event where mentees spend 5 minutes interviewing each mentor using a prepared set of question and then rating the encounters. The mentoring facilitator or task force uses these ratings to match mentors and mentees. Although assigning one mentor to an individual mentee tends to be the model, several universities have collaborative mentoring. For example, each new faculty at the UPenn (2014) has a mentoring team that includes the mentor/mentee dyad, the department chair, one or more senior nursing faculty, and a faculty, who is outside the department or university. Clearly, appropriate matching of the mentor/mentee dyads is foundational to an effective mentorship program. However, defining the relationship purpose and goals is an important next step.

Setting clear mentorship purpose and goals. Having a defined purpose to the mentoring relationship and setting goals for the new faculty's growth as an educator are critical in the first year of teaching. Without a clear purpose and goals, new faculty may feel lost in how to best access their mentor (Cangelosi, 2014; Gardner, 2014). Mutually identifying areas of growth is a first step in this process. Collaborative assessment of new faculty learning needs in an initial meeting with their mentor can guide the setting of goals and define the purpose of the mentoring relationship (Blauvelt \& Spath, 2008; COD, 2014; McAllister et al., 2014). A self-assessment survey such as the 
one used by Baker (2010) could be used to help new faculty identify key focus areas for growth as an educator. This same assessment can also be completed at the end of the academic year to evaluate new faculty growth and outcomes of the formal mentoring relationship (Baker, 2010).

The purpose and goals of the mentoring relationship can be formalized through completing a mentoring partnership agreement. These documents can include norms for the relationship such as maintaining confidentiality and commitment to meeting; new faculty and mentor expectations for the relationship; and mutually agreed upon goals (UNC Chapel Hill, 2014; UCSF, 2014). The agreement can then be evaluated at the end of the year (UCSF, 2014) or at 3 month intervals throughout the year (UNC Chapel Hill, 2014). The length of the mentorship partnership depends upon its purpose. If the purpose is to launch faculty in their first year, the mentee/mentor dyad can meet together for 2 to 3 semesters (Blauvelt \& Spath, 2008; COD, 2014; Slimmer, 2012). If the purpose is early career development, the mentorship relationship may extend beyond the first year (UCSF, 2014; UPenn, 2014). Once the mentor and mentee define their purpose and goals, intentional steps must be taken to solidify the mentoring relationship.

Solidifying the dyad relationship. A meaningful mentor-mentee relationship is characterized by "connectedness and inclusion, and communication and openness" (White et al., 2010, p. 603). It takes time spent in intentional dialogue to solidify the mentoring relationship into a meaningful one (Cangelosi, 2014; McAllister et al., 2014; Sawatzsky \& Enns, 2009; White et al., 2010; Wilson et al., 2010). Some mentoring programs require formal checkpoints that are identified in the mentoring partnership agreement. Formal checkpoints range from twice a semester (UNC Chapel Hill, 2014; UPenn, 2014) to monthly or weekly during the first semester and less often in subsequent semesters (Blauvelt \& Spath, 2008; COD, 2014; Santistiban \& Egues, 2014).

How the mentor and new faculty member spend this designated time is crucial to solidifying the relationship (McAllister et al., 2014). To facilitate communication, the UCSF (2014) mentoring program recommends that dyads use the 10/20/60 rule, which allots 10 minutes for personal and/or professional check-in; 20 minutes to discuss front burner issues; and 60 minutes for goal discussion. The new faculty and mentor need to also identify topics which are off limits for discussion. Other strategies for solidifying the mentoring relationship are to keep a mentoring journal of each meeting, which becomes a record of the mentoring relationship (UCSF, 2014; White et al., 2010; Wilson et al., 2010), or to complete a teaching/learning project together (McAllister et al.,2014). Sometimes dyad relationships do not solidify and mentoring programs need strategies to graciously end the relationship. For example, UNC Chapel Hill (2014) has a no fault opt out policy for mentors and mentees. The mentoring facilitator or mentorship team at UCSF (2014) and UPenn (2014) annually meet with new faculty to evaluate whether or not a new mentor is needed.

Integrating new faculty into the academic culture. Successful integration of new faculty into the academic culture is critical to their job satisfaction and retention (Baker, 2010). Mentors play a critical role in helping their mentees navigate the complexities of the faculty role and the individual educational institution. A structured orientation as described above lays the foundation for this process (Santisteban \& Egues, 2014). However, new faculty need ongoing guidance as they encounter their institution's expectations related to teaching, scholarship, and service (Wilson et al., 2010). Creating a welcoming community of nurse educators that allows a gradual acclimatization to faculty role responsibilities can mitigate some of the stresses of the first year of teaching (Cangelosi, 2014; Schoening, 2013; White et al., 2013). Whether communities meet weekly the first semester (Blauvelt \& Spath, 2008), monthly (Baker, 2010), or online (McAllister et al., 2014), their purpose is to provide a safe environment for new faculty to discuss teaching, faculty role responsibilities, and their experience of the transition from expert clinician to novice educator (Baker, 2010; Blauvelt \& Spath, 2008; McAllister et al., 2014). Clinicians often find themselves unprepared for setting teacher-student relationship boundaries and experiencing negative encounters with students (Schoening, 2013). Using simulation to practice managing difficult student scenarios within the safety of the community can also be effective (Hunt et al., 2013; Reid et al., 2013). Mentors facilitate this integration process through their advocacy and guidance.

Advocating and guiding the new faculty. The mentor plays a crucial role in guiding new faculty through the first year of teaching (Jacobsen \& Sherrod, 2012). Because teaching nursing skills to students is very different from performing these skills as an expert clinician, mentors can serve as guides as their mentees become teachers (Jacobsen \& Sherrod, 2012). Based on the individual needs of their mentees, topics for dialogue might include managing multiple faculty roles (Reid et al., 2013), navigating the unwritten rules that exist in all organizations 
(Wilson et al., 2010), using pedagogical resources, and accessing faculty development opportunities offered by their university or college, nursing education conferences, or online (Jacobsen \& Sherrod, 2012; Slimmer, 2012). Strategies that can build new faculty's confidence include opportunities to observe their mentors teach a course or plan a clinical day and to receive oral or written feedback about their own teaching and classroom/clinical management (Blauvelt and Spath, 2008; Gardner, 2014). Conducting a joint teaching/learning project with one’s mentor is another strategy discussed in the literature (McAllister et al., 2014; Sawatzky \& Enns, 2009). Because student evaluations can be devastating to a new faculty, Slimmer (2012) recommends that mentors meet with their mentees to review and interpret the previous semester's student course evaluations. Finally, to successfully implement a formal mentoring process, institutional resources need to be mobilized.

Mobilizing institutional resources. Administrative support for mentoring is critical to developing effective programs for new faculty. Mentoring programs need to be structured and formally acknowledged as core to a school's mission and vision (Cangelosi, 2014; Sawatzky \& Enns, 2009). Institutional resources that facilitate an effective mentoring program include time, money, mentor training, and administrative involvement. Time is a significant barrier to effective mentoring; providing faculty workload credits or release time can overcome this barrier (Schoening, 2013; UNC Chapel Hill, 2014). In addition, mentors could be paid a stipend (Baker, 2010). Funding joint mentee/mentor projects and workshops that prepare potential mentors for their role is an important investment in supporting new faculty (Schoening, 2013; Sawatzky \& Enns, 2009). A designated mentorship facilitator or task force appointed by the department chair can help sustain an effective mentoring program through preparing mentors, matching mentor/mentee dyads, and providing faculty development for mentees (Baker, 2010; UCSF, 2014; UNC Chapel Hill, 2014; UPenn, 2014).

The findings from this integrated literature review support the importance of a comprehensive program to support clinicians as they transition to the academic environment. These potential educators need resources for learning to teach, a welcoming community of nurse educators to help them feel comfortable in the academic environment, and mentors who can guide them as they master the faculty role. The literature offers helpful strategies that nursing education programs can incorporate into developing a process for supporting new faculty.

\section{Recommendations for Nursing Education}

Five recommendations were developed from this integrative literature review. The first recommendation is to create a sustainable infrastructure that includes the following steps: 1) identify and appoint a new faculty mentoring coordinator or team with primary responsibilities to develop and oversee the program; 2) create a mentoring toolkit (See Table 2 for a recommended outline); 3) determine expectations for how often mentors should meet with their mentees; 4) solicit and screen volunteer faculty to become mentors; 5) provide mentorship training through the nursing department, college, or university; 6) establish a mechanism for matching mentors with new faculty, such as a needs and interest inventory or speed mentoring event; 7) develop a process for evaluating the mentor/mentee relationship; 7) offer incentives for mentors such as faculty workload credit and/or stipends; and 8) plan celebrations that welcome new faculty and recognize mentors. The NLN Mentoring of Nursing Faculty Toolkit is recommended as an excellent resource that uses a series of questions to stimulate thinking about how to develop faculty mentoring programs (NLN, 2008). The university and college toolkits identified in this literature review can also provide ideas for development of a comprehensive mentoring program.

The second recommendation is that administration provide funds for new faculty to enroll in didactic courses for clinical faculty, such as the online courses offered by the CNL (2014) and IUSON (2014). The third recommendation is that regular mentor/mentee meetings be scheduled throughout the first academic year, such as at the beginning of each semester to set and review goals, 2 to 3 weeks after start of semester to review student evaluations from the previous semester, at semester mid-term, and at the conclusion of the school year. Fourth, it is recommended that the mentoring relationship focus on navigating the educational institution, resolving student issues, identifying supports needed for teaching, and managing the new faculty transition experience. The final recommendation focuses on the need for more research into the mentoring process. Much of the literature was descriptive, either qualitative studies of the mentoring experience or descriptions of mentoring programs. Clearly more research is needed to evaluate the mentoring process. 


\section{Conclusions}

Structured, formal, comprehensive mentoring programs are essential to the future of nursing education in order to recruit and retain qualified clinicians in academic nursing education. Sustainable mentoring programs require recognition of mentoring as central to nursing education and administrative investment of resources. These resources include appointment of a mentoring facilitator or mentoring team, funding for new faculty development related to teaching, ongoing orientation to the academic environment, mentor training with clear guidelines about the mentor/mentee relationship, and tools to evaluate program outcomes.

\section{References}

Anderson, J. K. (2009). The work-role transition of expert clinician to novice academic educator. Journal of Nursing Education, 48(4), 203-208.

Baker, S. L. (2010). Nurse educator orientation: Professional development that promotes retention. The Journal of Continuing Education in Nursing, 41, 413-417. doi:10.3928/00220124-20100503-02

Berk, R. A. (2010). Where's the chemistry in mentor-mentee academic relationships? Try speed mentoring!. The International Journal of Mentoring and Coaching, 8, 85-92.

Blauvelt, M. J., \& Spath, M. L. (2008). Passing the torch: A faculty mentoring program at one school of nursing. Nursing Education Perspective, 29, 29-33.

Cangelosi, P. R. (2014) Novice nurse faculty: In search of a mentor. Nursing Education Perspectives, 35, 327-329. http://dx.doi.org/10.5480/13-1224

Cangelosi, P. R., Crocker, S., \& Sorrell, J. M. (2009). Expert to novice: Clinicians learning new roles as clinical nurse educators. Nursing Education Perspectives, 30, 367-371.

College of Dupage. (2013). Nursing department mentor/mentee toolkit. Retrieved from: http://www.cod.edu/programs/nursing/pdf/mentor_mentee_toolkit.pdf

Connecticut League for Nursing. (2014). Clinical faculty course. Retrieved from http://www.ctleaguefornursing.org/online-education-clinical-faculty

Gardner, S. S. (2014). From learning to teach to teaching effectiveness: Nurse educators describe their experiences. Nursing Education Perspectives, 35, 106-111. doi:10.5480/12-821.1

Hunt, C. W., Curtis, A. M., \& Sanderson, B. K. (2013). A program to provide resources and support for clinical associates. The Journal of Continuing Education in Nursing, 44, 269- 373. doi:10.3928/0022012420130402-27

Indiana University School of Nursing. (2014). Clinical faculty: A new practice role. Retrieved from http://nursing.iu.edu/development/online-courses/clinical-faculty.shtml

Jacobsen, S. L., \& Sherrod, D. R. (2012). Transformational mentorship models for nurse educators. Nursing Science Quarterly, 25, 279-284. doi:10.1177/0894318412447565

McDermid, F., Peters, K., Jackson, D., \& Daly, J. (2012). Factors contributing to the shortage of nurse faculty: A review of the literature. Nursing Education Today, 32(5), 65-69. doi: 10.1016/j.nedt.2012.01.011.

McAllister, M., Oprescu, F., \& Jones, C. (2014). Envisioning a process to support transition from nurse to educator. Contemporary Nurse, 46, 242-250.

National League for Nursing. (2006). Position statement: Mentoring of nurse faculty. Retrieved from http://www.nln.org/aboutnln/PositionStatements/index.htm

National League for Nursing. (2008). The Mentoring of Nursing Faculty Tool Kit@. Retrieved from http://www.nln.org/facultyprograms/MentoringToolkit/

Nick, J. M., Delahoyde,T. M., Prato,D. D., Mitchell, C., Ortiz, J., Ottley, C., ... Siktberg, L. (2012). Best practices in academic mentoring: A model for excellence. Nursing Research and Practice, 2012, 1-9. doi:10.1155/2012/937906

Reid, T. P., Hinderer, K. A., Jarosinski, J. M., Mister,B. J., \& Seldomridge, L. A. (2013). Expert clinician to clinical teaching: Developing a faculty academy and mentoring initiative.

Nurse Education in Practice, 13, 288-293. doi:10.1016/j.nepr.2013.03.022 Santisteban, L., \& Egues, A. L. (2014). Cultivating adjunct faculty: Strategies beyond orientation. Nursing Forum, 49, 152-158. doi:10.1111/nuf.12106

Sawatzky, J. V., \& Enns, C. L. (2009). A mentoring needs assessment: Validating mentorship in nursing education. Journal of Professional Nursing, 25, 145-150. doi:10.1016/j.profnurs.2009.01.003

Schoening, A. M. (2013). From bedside to classroom: The Nurse Educator Transition Model. Nursing Education Perspective, 34, 167-172. 
This is an author-produced, peer-reviewed version of this article. The final, definitive version of this document can be found online at Journal of Nursing Education, published by Slack, Inc. Copyright restrictions may apply. doi: 10.3928/01484834-20150617-01

Schriner, C. L. (2007). The influence of culture on clinical nurses transitioning into the faculty role. Nursing Education Perspectives, 28(4), 145-149.

Siler, B. B., \& Kleiner, C. (2001). Novice faculty: Encountering expectations in academia. Journal of Nursing Education, 40(9), 397-403.

Slimmer, L. (2012). A teaching mentorship program to facilitate excellence in teaching and learning. Journal of Professional Nursing, 28, 185-185. doi:10.1016.j.profnurs.2011.11.006

University of California, San Francisco. (2012). Faculty mentoring program. Retrieved from: http://academicaffairs.ucsf.edu/ccfl/faculty_mentoring_program_resources.php

University of North Carolina at Chapel Hill. (2008). School of nursing faculty mentoring program. Retrieved from: https://sonapps.unc.edu/faculty/fdsc/mentoring.html

University of Pennsylvania. (2014). Faculty mentorship program. Retrieved from: http://www.nursing.upenn.edu/mentorship/Pages/FacultyMentorshipProgram.aspx\#facult y-mentor

White, A., Brannan, J., \& Wilson, C. B. (2010). A mentor protégé program for new faculty, part 1: Stories of protégés. Journal of Nursing Education, 49, 601-607. doi:10.3928/01484834-20100630-0

Wilson, C. C., Brannan, J., \& White, A. (2010). A mentor protégé program for new faculty, part 2: Stories of mentors. Journal of Nursing Education, 49, 665-671. doi:10.3928/01484834-20100730-08 